\title{
Desempenho de repetições e tempo sob tensão no método cluster sets vs. tradicional em uma sessão de treinamento de força
}

\author{
Repetition performance and time under tension of cluster \\ sets vs. traditional in a resistance training session
}

\section{Anderson Correa Dantas ${ }^{1}$ \\ Juan Câmara Pinheiro² \\ Marianna de Freitas Maia ${ }^{3}$ \\ Gabriel Andrade Paz ${ }^{4}$ \\ Igor Nasser ${ }^{5}$ \\ Humberto Miranda ${ }^{6}$}

\section{Endereço para correspondência:}

Igor Nasser

Universidade Federal do Rio de Janeiro - UFRJ Avenida Carlos Chagas, 540 - Cidade Universitária 21941-590 - Rio de janeiro - RJ [Brasil] igor_nasser@hotmail.com
1 Programa de Pós-graduação Lato Sensu em Treinamento de Força, Escola de Educação Física e Desportos, Universidade Federal do Rio de Janeiro - UFRJ. Rio de Janeiro, RJ - Brasil. ORCID: https://orcid.org/0000-0001-6348-1866

2 Programa de Pós-graduação Lato Sensu em Treinamento de Força, Escola de Educação Física e Desportos, Universidade Federal do Rio de Janeiro - UFRJ. Rio de Janeiro, RJ - Brasil. ORCID: https://orcid.org/0000-0002-2921-0306

3 Programa de Pós-graduação Stricto Sensu em Educação Física, Escola de Educação Física e Desportos, Universidade Federal do Rio de Janeiro - UFRJ. Rio de Janeiro, RJ - Brasil. ORCID: https://orcid.org/0000-0001-8284-6533

4 Programa de Pós-graduação Stricto Sensu em Educação Física, Escola de Educação Física e Desportos, Universidade Federal do Rio de Janeiro - UFRJ. Rio de Janeiro, RJ - Brasil. ORCID: https://orcid.org/0000-0001-8474-9753

5 Programa de Pós-graduação Stricto Sensu em Educação Física, Escola de Educação Física e Desportos, Universidade Federal do Rio de Janeiro - UFRJ. Rio de Janeiro, RJ - Brasil. ORCID: https://orcid.org/0000-0003-3116-393X

6 Departamento de Ginástica, Escola de Educação Física e Desportos, Universidade Federal do Rio de Janeiro - UFRJ Rio de Janeiro, RJ, Brasil.

ORCID: https://orcid.org/0000-0001-7911-3910

\begin{abstract}
Resumo
Introdução: O método cluster sets (CLS) surge como um método de treinamento de força (TF) incluindo pequenos intervalos dentro da série. Objetivo: comparar o desempenho agudo do método CLS e o método tradicional (TRAD). Métodos: participaram do estudo 14 homens com experiência em TF. Os participantes realizaram dois protocolos distintos: TRAD - três séries de oito repetições com $90 \%$ de 8 repetições máximas (RM) e intervalo de dois minutos; CLS - três séries com oito repetições com $90 \%$ de 8 RM, e 30 segundos de intervalo a cada duas repetições. Resultados: o método CLS foi capaz de manter o desempenho ao longo das séries, sem reduções significativas nas séries 2 e 3, em ambos exercícios. Ao compará-lo com o método TRAD, o número de repetições realizadas foi significativamente maior $(p \leq 0,05)$ para o CLS. Conclusão: o CLS proporcionou desempenho de repetições máximas superior em comparação ao TRAD. Não foram verificadas diferenças no tempo sob tensão entre os protocolos.
\end{abstract}

Descritores: Treinamento de Resistência. Exercício. Desempenho.

\begin{abstract}
Introduction: Cluster sets (CLS) comes up a resistance training (RT) method that includes short intraset rest intervals. Objective: investigate acute performance in multiple sets in the CLS method vs. traditional (TRAD) Methods: 14 men trained in RT participated in this study. Subjects performed two distinct protocols: TRAD - three sets of eigth repetitions with $90 \%$ of 8 repetition maximum (RM) and two minutes rest inteval; CLS - three sets of eigth repetitions with $90 \%$ of eigth repetitions, with intraset rest of 30 seconds every two repetitions. Results: CLS method was able to maintain performance throughout sets, without significant reductions in sets 2 and 3, in both exercises. Compared to TRAD, total repetitions performed were significantly higher than for CLS than TRAD $(p \leq 0,05)$. Conclusion: CLS provided greater total repetitions performed compared to TRAD. No significant differences were observed for time under tension between protocols.
\end{abstract}

Keywords: Resistance Training. Exercise. Performance. 


\section{Introdução}

O treinamento de força (TF) é a principal modalidade de exercício físico quando o objetivo é o desenvolvimento de adaptações musculares como força, resistência e hipertrofia ${ }^{1}$. Sua prescrição consiste na utilização de variáveis de treinamento que são manipuladas ao longo de um período como um processo de periodização ${ }^{2}$. Sabe-se que a manipulação de variáveis como, volume, intensidade, e intervalo entre séries, de forma sistemática, pode aperfeiçoar os resultados ${ }^{3}$. Tradicionalmente, o TF é prescrito em séries, com zona de repetições determinada, sendo separadas por um intervalo inter-séries ${ }^{4}$.

Recentemente, criaram-se variações no modelo de execução das séries, com o seu fracionamento e a utilização de um breve intervalo intra-série, conhecido como método cluster sets $^{5}$. O resultado da combinação de variáveis originam os métodos de treinamento, sendo a manipulação do formato das séries uma possibilidade. $\mathrm{O}$ método cluster sets surge como estratégia para aperfeiçoar o desempenho de potência em uma sessão de TF, uma vez que o modelo tradicional leva o praticante à fadiga no final da série, resultando em uma redução na velocidade de execução e produção de potência ${ }^{6}$. Desse modo, esse intervalo dentro da série permite uma recuperação momentânea, que permite a manutenção da velocidade durante toda a série. Outra vantagem adicional desse método é a possibilidade do aumento do volume de treinamento, a partir de um maior número de repetições executadas na sessão, o qual indicaria um maior desempenho desse método comparado ao modelo tradicional de prescrição ${ }^{7}$.

Apesar de algumas evidências indicando os potenciais benefícios do método cluster sets, as diferentes configurações que são permitidas dificultam afirmar qual modelo de prescrição mais adequado para determinado objetivo. $\mathrm{O}$ seu formato permite a inclusão de múltiplos intervalos dentro de uma série, podendo ser realizado até o modelo de intervalo entre repetições ${ }^{8}$. Desse modo, acredita-se que dentro de uma prescrição de treinamento, esse método favoreceria a manutenção do desempenho, a partir de uma menor redução de repetições ao longo de múltiplas séries. A prescrição de um treinamento que apresentasse um maior volume total de treinamento (VTT) na sessão indicaria um maior tempo sob tensão (TST) o qual a músculo é submetido. Tanto o VTT quanto o TST parecem importantes para os diferentes processos de adaptações musculares ${ }^{9,10}$.

Portanto, o objetivo do presente estudo foi investigar a capacidade de manutenção do desempenho ao longo de múltiplas séries do método cluster sets em comparação ao método tradicional em uma sessão de exercícios para membros superiores. Como parâmetro de desempenho, foi quantificado o desempenho de repetições em cada série e o TST, a partir da medição em segundos para cada série nos exercícios supino reto barra longa (SR) e puxada aberta (PU).

\section{Materiais e métodos}

\section{Amostra}

Participaram do estudo 14 homens com experiência mínima de seis meses em TF. Para serem incluídos no estudo, os participantes deveriam ser saudáveis e idade entre 20 e 35 anos. Todos os selecionados tinham como rotina de TF uma frequência de 4-5 dias na semana, em sessões que duravam de 50-60 minutos e estavam engajados em um programa com o objetivo de hipertrofia muscular. A prescrição seguia recomendações tradicionais para esse objetivo, com múltiplas séries por exercícios, com uma divisão em split routine, sendo um programa para membros superiores e outro para inferiores, com uma zona de repetição intermediária, de 8 a 12 repetições e intervalos de 1-2 minutos entre séries ${ }^{11,12}$. As informações em relação a caracterização da amostra estão presentes na Tabela 1. Foram excluídos do estudo aqueles que apresentavam alguma lesão osteomioarticular de membros superiores que impedisse a realização dos exer- 
cícios aplicados neste estudo e alguma resposta afirmativa no questionário Physical Activity Readiness Questionnaire (PAR-Q). Como aspecto ético, foram adotadas normas da participação em pesquisa envolvendo seres humanos, conforme a Resolução 466/2012 do Conselho Nacional de Saúde, para a realização do teste. Além disso, todos os participantes assinalaram um Termo de Consentimento Livre e Esclarecido (TCLE) para participação na pesquisa, contendo informações de todos os processos que seriam adotados ao longo do estudo.

Para a realização das medidas antropométricas, foi utilizado um estadiômetro profissional Sanny®(modelo ES2020, São Paulo, Brasil) com capacidade máxima para 2,10m e acurácia de $0,001 \mathrm{~m}$. Para aferição da composição corporal (massa corporal total - MCT) foi realizada utilizando uma balança elétrica de bioimpedância (BIA) da marca InBody ${ }^{\circledR}$ (modelo 230 Biospace, Miami, EUA) com limitação de $250 \mathrm{~kg}$ e precisão de $100 \mathrm{~g}$. ção do método de treinamento. A figura 1 apresenta o fluxograma do estudo e os procedimentos adotados.

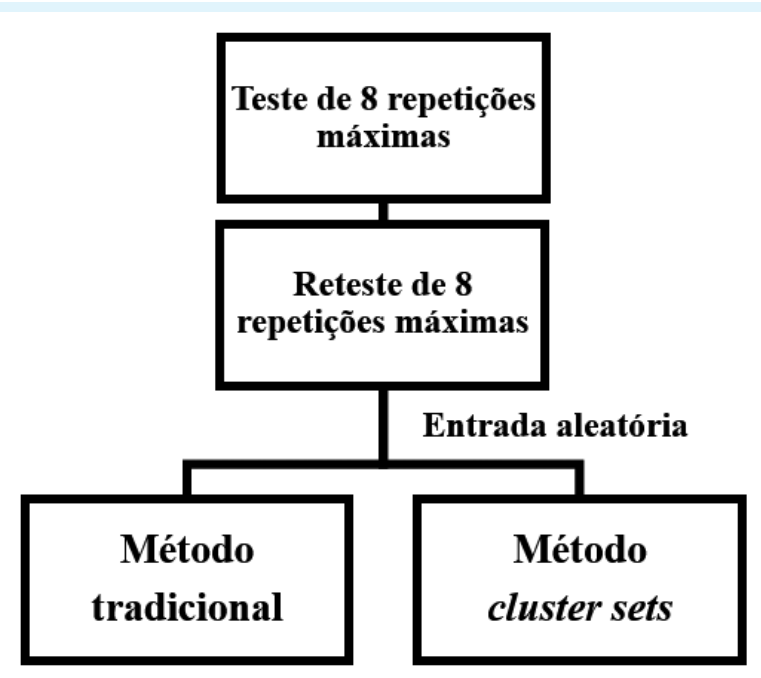

Figura l: Fluxograma do estudo Fonte: Os Autores.
Tabela l: Caracterização da amostra

\begin{tabular}{c|c|c|c|c|c}
\hline & Idade & Estatura & Peso & IMC & MLG \\
\hline Média (DP) & $26,1(4,3)$ & $1,76(0,04)$ & $81,4(9,1)$ & $26,19(2,41)$ & $80,7(9,5)$ \\
\hline
\end{tabular}

Legenda: DP - desvio padrão; IMC - índice de massa corporal; MLG massa livre de gordura

Fonte: Os Autores.

\section{Procedimentos}

O estudo consistiu de um ensaio clínico randomizado, com entrada contrabalanceada e de corte transversal. Para conclusão do estudo, foram realizadas quatro visitas separadas por intervalos de 48 horas. Na primeira visita, os participantes tinham acesso às informações mais precisas em relação ao estudo e assinalavam o questionário PAR-Q e o TCLE. Em seguida, realizaram as medidas antropométricas e foram submetidos ao teste de oito repetições máximas (8 RM). Na segunda visita, os voluntários realizaram o re-teste de $8 \mathrm{RM}$. Na terceira e quarta visita, os participantes realizaram dois protocolos distintos de $\mathrm{TF}$, com manipula-
Determinação da carga de oito repetições móximas (8 RM)

Instruções padronizadas foram fornecidas antes do teste, de modo que o avaliado estivesse ciente de toda a rotina que envolveu a coleta de dados. O teste para obtenção da sobrecarga de 8 RM foram determinadas para cada sujeito nos exercícios supino reto barra longa (SR) e puxada aberta (PU), seguindo as orientações propostas por Paz et al..$^{13}$. O avaliado foi instruído sobre a técnica de execução do exercício previamente a aplicação do teste. A sobrecarga inicial para o teste de $8 \mathrm{RM}$ foi o mesmo que o voluntário costumava utilizar nos seus treinos diários. Foram utilizadas até três tentativas para determinar a carga para $8 \mathrm{RM}$ em cada um dos exercícios. Foram fixados intervalos entre as tentativas entre três e cinco minutos para cada exercício, e um intervalo entre exercícios de 10 minutos. Os partici- 
pantes foram orientados a realizar a velocidade de execução habitual de treinamento. Durante o teste, o avaliador esteve atento à posição adotada pelo praticante, levando a interpretações errôneas dos escores obtidos. Estímulos verbais foram realizados com o intuito de manter o nível de motivação elevado ${ }^{14}$. Após um período de 48 horas do teste, foi realizado um reteste seguindo as mesmas orientações do teste, com o intuito de aperfeiçoar a precisão da carga de $8 \mathrm{RM}$. Todas as sessões de teste e reteste foram aplicados pelos mesmos avaliadores, com o intuito de minimizar potenciais interferências.

O exercício SR (Hammer, USA) utilizava uma barra olímpica inox com um comprimento: $2,20 \mathrm{~m}$ e peso de $20 \mathrm{~kg}$ com resistência de até $600 \mathrm{~kg}$. A barra maciça cromada é confeccionada, com pegas recartilhadas e batentes de anilha. O movimento iniciava com os braços estendidos e deveria ser flexionado até que a barra se aproximasse do tórax. Em seguida, os participantes deveriam estender o cotovelo retornando a barra para a posição inicial. No exercício PU (TechnoGym LatPulldown, USA) com medidas de $2285 \times 845 \times 1220 \mathrm{~mm}$ e tem uma pilha de peso padrão de $100 \mathrm{~kg}$ e uma pilha de peso pesado de 130 quilogramas. O movimento iniciava com os braços abertos em uma barra reta, flexionando o cotovelo na fase concêntrica do movimento. Durante a fase excêntrica, o participante retornaria a posição inicial até completa extensão dos braços.

\section{Protocolos Experimentais}

No terceiro e quarto dia, foram aplicados os protocolos experimentais, com entrada contrabalanceada entre os métodos e intervalo de 48 horas entre as sessões. No método tradicional (TRAD) foram realizadas três séries de oito repetições com $90 \%$ de 8 RM e intervalo de dois minutos. No método cluster sets (CLS) foram realizadas três séries com oito repetições com 90\% de $8 \mathrm{RM}$, sendo que o intervalo entre séries foi fracionado para intervalo intra-série, onde a cada duas repetições eram dados 30 segundos de intervalo (Figura 2). Dessa forma, cada exercício foi equalizado pelo tempo total de intervalo. Entre exercícios foram dados três minutos de intervalo em ambos os métodos. Os participantes foram orientados a atingir oito repetições em todas as séries, e por conta disso, foi adotada a sobrecarga de $90 \%$ do obtido no teste de $8 \mathrm{RM}$. Para verificar a capacidade de manutenção do desempenho ao longo das séries, foi utilizado o total de repetições realizadas e o TST de cada série, medido a partir de um cronômetro digital do início da realização da série até o momento de falha muscular.

\section{Tratamento estatístico}

O tratamento estatístico foi realizado no software SPSS versão 20.0 (Chicago, IL, USA). A análise estatística foi realizada inicialmente utilizando o teste Shapiro-Wilk de normalidade e teste de homocedasticidade (critério Bartlett). Todas as variáveis apresentaram distribuição normal e homocedasticidade. O coeficiente de correlação intraclasse $\left(\mathrm{CCI}=\left(\mathrm{MS}_{\mathrm{b}}-\mathrm{MS}_{\mathrm{w}}\right) /\left[\mathrm{MS}_{\mathrm{b}}\right.\right.$ $+(\mathrm{k}-1) \mathrm{MS}_{\mathrm{w}}$ ) foi calculado para verificar a reprodutibilidade do teste e re-teste de 8RM nos exercícios supino reto e puxada aberta. A two-wayANOVA para medidas repetidas seguida do post hoc de Bonferroni foi aplicado para determinar se ocorreu diferença significativa ou interação entre o tipo de método treinamento e entre as séries (1-3) relacionado ao desempenho de repetições e tempo sob tensão. O valor de $\mathrm{p}<0,05$ foi adotado para todas as análises inferenciais.

\section{Resultados}

Quanto ao desempenho de repetições máximas no exercício SR foi verificada diferença significativa entre os métodos $(\mathrm{F}=$ 283,23; $\mathrm{p}=0.0001)$ e séries $(\mathrm{F}=66.91 ; \mathrm{p}=0.0001)$. Adicionalmente, foram verificadas interações entre os métodos e séries ( $\mathrm{F}=86.56 ; \mathrm{p}=0.0001)$. A capacidade de manutenção de desempenho de repetições reduziu significativamente entre as 
Tradicional

\begin{tabular}{|l|l|l|l|l|l|l|l|l|l|}
\hline Rep 1 & $\operatorname{Rep} 2$ & $\operatorname{Rep~3}$ & $\operatorname{Rep} 4$ & $\operatorname{Rep} 5$ & $\operatorname{Rep} 6$ & $\operatorname{Rep} 7$ & $\operatorname{Rep} 8$ \\
\hline
\end{tabular}

\section{Cluster 1}

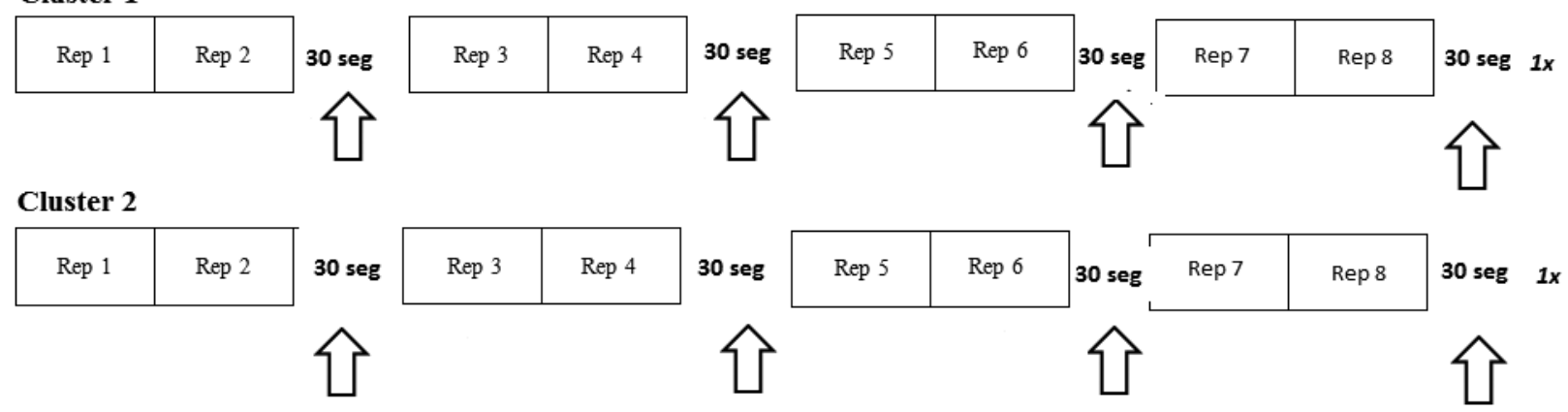

Cluster 3

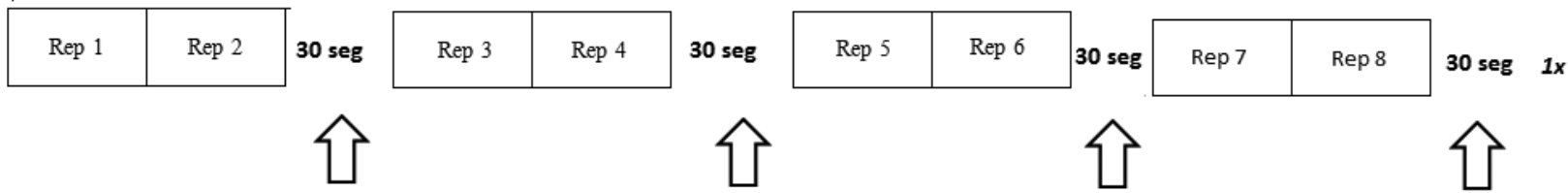

Figura 2: Esquematização do método tradicional e das três séries do método cluster

Fonte: Os Autores.

séries 1 versus série $2(p=0.0001)$ e $3(p=0.0001)$, e entre as séries 2 e 3 ( $p=0.0001)$ no TRAD. Todavia, no método CLS não houve diferença entre as séries. Por outro lado, o desempenho de repetições foi superior nas séries $2(\mathrm{p}=0.0001) \mathrm{e}$ $3(\mathrm{p}=0.0001)$ no método CLS versus TRAD.

Em relação ao desempenho de repetições máximas na PU, foi verificada diferença significativa entre os métodos $(F=166,97$; $\mathrm{p}=0.0001)$ e séries $(\mathrm{F}$ $=68.64 ; \mathrm{p}=0.0001)$. Adicionalmente, foram verificadas interações entre os métodos e séries $(\mathrm{F}=86.86 ; \mathrm{p}=$ 0.0001). A capacidade de manutenção de desempenho de repetições reduziu significativamente entre as séries 1 versus série $2(\mathrm{p}=$ $0.0001)$ e $3(p=0.0001)$, e entre as séries 2 e 3 ( $\mathrm{p}=0.0001)$ no TRAD. No CLS, não houve diferença entre as séries. Por outro lado, o desempenho de repetições foi superior nas séries $2(\mathrm{p}=0.0001)$ e 3 ( $\mathrm{p}=0.0001)$ no método CLS versus TRAD. Os resultados de desempenho de repetições nos diferentes métodos nos exercícios SR e PU estão presentes na figura 3.

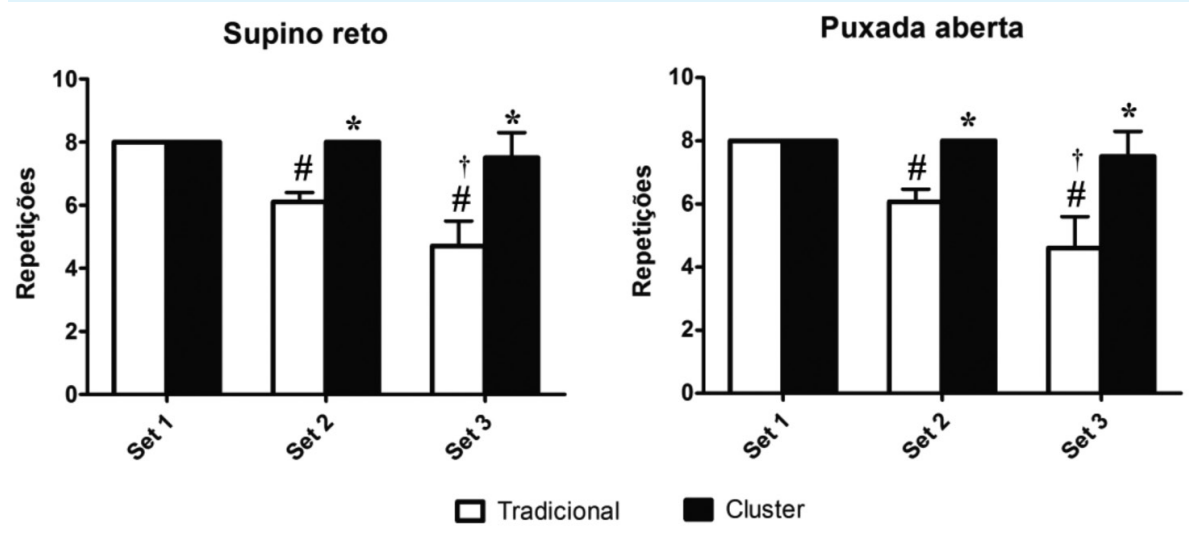

Figura 3: Desempenho de repetições no supino reto e puxada aberta. \# Diferença significativa intra-protocolo para série $1(p \leq 0,05)$; † Diferença significativa intra-protocolo para série $2(p \leq 0,05)$; Diferença significativa para o método tradicional $(p \leq 0,05)$. Fonte: Os Autores. 
Quanto ao TST no exercício SR, não foi verificada diferença significativa entre os métodos e séries (Figura 2). Todavia, foram verificadas interações entre os métodos e séries $(\mathrm{F}=11,41 ; \mathrm{p}=$ 0.0001). Em relação ao TST no exercício puxada aberta, não foi verificada diferença significativa entre os métodos e séries. Todavia, foram verificadas interações entre os métodos e séries ( $F$ = 9.364; $\mathrm{p}=0.0001)$. Os resultados de TST dos diferentes métodos nos exercícios SR e PU estão presentes na figura 4 .

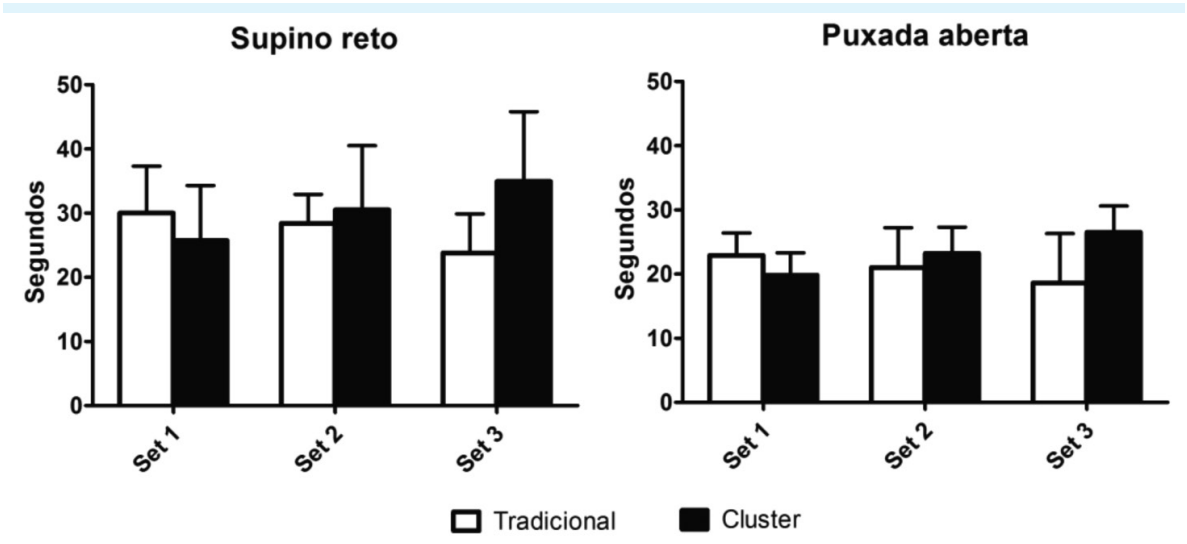

Figura 4: Tempo sob tensão (segundos) no supino reto e puxada aberta

Fonte: Os Autores.

\section{Discussão}

O presente estudo evidenciou que o método CLS foi capaz de manter o desempenho ao longo das séries, sem reduções significativas nas séries 2 e 3, tanto para o exercício SR quanto para a PU. Ao compará-lo com o método TRAD, o número de repetições realizadas foi significativamente maior, indicando que o método CLS é uma boa estratégia para manutenção do volume no TF. Apesar desses achados, o TST não mostrou diferença entre os métodos em nenhuma das séries e exercícios.

Os achados não surpreendem em relação ao melhor desempenho de repetições para o CLS, uma vez que os intervalos intra-séries permitem recuperações parciais com remoção de resíduos que promovem a fadiga, bem como a ressíntese do ATP e dos fosfatos de creatina ${ }^{4}$. Estudos anteriores ${ }^{7,15,16}$ evidenciaram que o método CLS é capaz de aumentar o VTT que é o produto do total de séries $\mathrm{x}$ soma de repetições $x$ sobrecarga $^{13}$. O maior VTT atribuído ao CLS em comparação ao TRAD pode se dar tanto pela capacidade de realizar maiores números de repetições $^{7}$ quanto pela necessidade de redução da sobrecarga pela incapacidade de atingir um determinado número de repetições estipulado ${ }^{14}$.

No presente estudo determinou-se que oito repetições deveriam ser alcançadas (90\% de 8-RM) em todas as séries dos exercícios, porém para verificar a capacidade de manutenção do desempenho, não foram realizadas alterações na sobrecarga, evidenciando um menor volume para o TRAD. Em um estudo que ajustou a sobrecarga para manutenção do desempenho, Oliver et al..$^{15}$ mostraram que na quarta série do agachamento livre, o método TRAD apresentou menor capacidade de manter o desempenho (10 repetições) ao compará-lo com o CLS ( 2 x 5 repetições e 30 segundos de intervalo intra-série). Em outro estudo, Tufano et al. ${ }^{5}$ analisaram a resposta metabólica e hormonal em oito indivíduos treinados há seis meses no agachamento em protocolos de CLS com equalização de carga e volume. Três protocolos de 36 repetições e 420 segundos de intervalo foram organizados em: cluster de quatro (CS4) com 30 segundos de intervalo a cada quatro repetições (após a 12a e $24^{a}$ repetição foram dados 120 segundos); nove séries de quatro repetições e 52,5 segundos de intervalo (RR4); e as 36 repetições com intervalo de 12 segundos intra-série (RR1). Em condições equalizadas não foram verificadas diferenças no hormônio do cresci- 
mento $(\mathrm{GH})$, testosterona livre e cortisol para nenhum dos protocolos.

Maiores volumes no TF têm sido atribuídos a melhores adaptações de força, hipertrofia e resistência muscular ${ }^{17,18}$. Treinamentos com maiores VTTs, a partir de um melhor desempenho de repetições, podem favorecer essas adaptações?. Desse modo, métodos que proporcionem manutenção do volume, como o CLS, podem contribuir nesses resultados. Apesar disso, o TUT não acompanhou os achados de repetições, apesar do maior volume. Menores sobrecargas são capazes de produzir movimentos mais rápidos e menor produção de força ${ }^{15}$. Entretanto, o método TRAD promove um acúmulo de resíduos ao longo das repetições, reduzindo a velocidade dos movimentos no final das séries ao compará-lo com protocolos $\mathrm{CLS}^{19}$.

Assim, o que justifica a ausência de diferença no TST apesar do maior volume do CLS são as reduções da velocidade do movimento que ocorrem no TRAD, especialmente nas repetições finais. Em contrapartida, o método CLS é conhecido por manutenção da velocidade do movimento ao longo das repetições. Em um estudo cujo objetivo foi verificar a cinética e a cinemática do agachamento livre no método CLS e no TRAD, Oliver et al. ${ }^{16}$ recrutaram 24 indivíduos do sexo masculino. O protocolo CLS foi realizado com quatro séries, sendo $2 \times 5$ repetições e intervalo intra-série de 30 segundos e intervalo entre séries de 90 segundos; enquanto que no TRAD foram realizadas quatro séries de 10 repetições e intervalo entre séries 120 segundos. Com sobrecarga de $70 \%$ de $1 \mathrm{RM}$, foram verificadas maiores médias de produção de força nas séries 2 e 4 nas repetições finais $(7,8,9$ e 10) no método CLS em comparação ao TRAD. Adicionalmente, maiores velocidades de movimentos foram identificadas nas repetições finais (6-10 repetições) para o método CLS, indicando que a manutenção da potência do movimento foi superior ao compará-lo com o TRAD. Corroborando com o presente estudo, Oliver et al. (2015b) identificou menores TST para o método CLS ao comparar séries e repetições isoladas do método TRAD. Aparentemente, es- sas respostas agudas se refletem de forma crônica, indicando que recuperações breves são boas estratégias nas adaptações de força e potência ${ }^{20}$. O estudo apresenta algumas limitações, como a amostra restrita de apenas 14 indivíduos, com experiência moderada em TF. Além disso, alguns métodos para produção de força e mensuração da velocidade dos movimentos não foram utilizados. Porém o estudo possui uma boa transferência para o ambiente prático do TF uma vez que os exercícios aplicados nas sessões são comumente utilizados na prescrição do TF. O estudo contribui para informações na elaboração da prescrição do TF, uma vez que os métodos apresentam estímulos diferentes, e a seleção deve ser feita de acordo com os objetivos estabelecidos.

\section{Conclusão}

Em conclusão, o CLS proporcionou desempenho de repetições máximas superior em comparação ao TRAD. Todavia, não se verificou diferença no TST entre os protocolos. Portanto, considerando as características metodológicas e ecológicas adotadas no presente estudo, o método CLS parece ser uma alternativa interessante para aumentar o VTT quando comparado ao TRAD.

\section{Referências}

1. Schoenfeld BJ, Peterson MD, Ogborn D, Contreras B, Sonmez GT. Effects of low- vs. high-load resistance training on muscle strength and hypertrophy in well-trained men. J of Strength and Cond Res 2015a;29(10):1954-1963.

2. Harries SK, Lubans DR, Callister R. Systematic review and meta-analysis of linear and undulating periodized resistance training programs on muscular strength. J of Strength and Cond Res 2015;29(4):1113-1125.

3. Williams TD, Tolusso DV, Fedewa MV, Esco MR. Comparison of periodized and non-periodized resistance training on maximal strength: a metaanalysis. Sports Med 2017;47(10):2083-2100. 
4. Tufano JJ, Brown LE, Haff G. Theoretical and practical aspects of different cluster sets structures: a systematic review. J of Stength and Cond Res 2016;31(3):848-867.

5. Tufano JJ, Conlon JA, Nimphius S, Oliver JM, Kreutzer A, Haff G. Different cluster sets results in similar metabolic, endocrine, and perceptual responses in trained men. J of Strength and Cond Res 2017; 33(2):346-354.

6. Nicholson G, Ispoglou T, Bissas A. The impact of repetitions mechanics on the adaptation resulting from strength-, hypertrophy- and clustertype resistance training. Eur J of Appl Physiol 2016;116(10):1875-1888.

7. Iglesias-Soler E, Carballeira E, Sánchez-Otero T, Mayo X, Fernández-del-Omo M. Performance of maximum repetitions with cluster-set configuration. Int J Sports Physiol Perform 2014;9(4):637-642.

8. Xian M, Eliseo IS, Derek KJ. Perceived exertion is affected by the submaximal set configuration used in resistance training. J of Strength and Cond Res 2017; 33(2):426-432.

9. Schoenfeld BJ, Pope ZK, Benik FM, Hester GM, Sellers J, Nooner JL, et al. Longer interest rest periods enhance muscle strength and hypertrophy in resistance-trained men. J of Strength and Cond Res 2015b;30(7):1805-1812.

10. Schoenfeld BJ, Ogborn DI, Krieger JW. Effect of repetition duration during resistance training on muscle hypertrophy: a systematic review and metaanalysis. Sports Med 2015;45(4):577-585

11. American College of Sports Medicine. Position stand: Progression models in resistance training for healthy adults. Medicine and Science in Sports Medicine 2011;42(3):11341359.

12. Schoenfeld BJ, Ratamess NA, Peterson MD, Contreras B, Tiryaki-Sonmez G. Influence of resistance training frequency on muscular adaptations in well-trained men. J of Strength and Cond Res 2015c;29(7):1821-1829.
13. Paz GA, Robbins DW, de Oliveira CG, Bottaro M, Miranda H. Volume load and neuromuscular fatigue during an acute bout of agonist-antagonist pairedset versus traditional-set training. J of Strength and Cond Res 2017;31(10):2777-2784.

14. Miranda H, Figueiredo T, Rodrigues B, Paz GA, Simão R. Influence of exercise order on repetition performance among all possible combinations on resistance training. Res Sports Med 2013;21(4):355366.

15. Oliver JM, Kreutzer A, Jenke S, Phillips MD, Mitchell JB, Jones MT. Acute response to cluster sets in trained and untrained men. Eur J of Appl Physiol 2015a;115(11):2383-2393.

16. Oliver JM, Kreutzer A, Jenke SC, Phillips MD, Mitchell MB, Jones MT. Velocity drives greater power observed during back squat using cluster sets. J of Strength and Cond Res 2015b;30(1):235-243.

17. Figueiredo VC, de Salles BF, Trajano GS. Volume for muscle hypertrophy and health outcomes: the most effective variable in resistance training. Sports Med 2018;48(3):499-505.

18. Radaelli R, Fleck SJ, Leite T, Leite RD, Pinto RS, Fernandes L, Simão R. Dose-response of 1, 3, and 5 sets of resistance training on strength, local muscular endurance, and hypertrophy. J of Strength and Cond Res 2015;29(5):1349-1358.

19. García-Ramos A, Padial P, Haff G, ArgüellesCienfuegos J, García-Ramos M, Conde-Pipó, et al. Effect of different interrepetition rest periods on barbell velocity loss during ballistic bench press exercise. J of Strength and Cond Res 2015;29(9):23882396.

20. Oliver JM, Jagim AR, Sanchez AC, Mardock MA, Kelly KA, Meredith HJ, et al. Greater gains in strength and power with intraset rest interval in hypertrophic training. J of Strength and Cond Res 2013;27(11):3116-3131. 